\title{
OVCA1: emerging as a bona fide tumor suppressor
}

\author{
Michael Rugaard Jensen and Kristian Helin ${ }^{1}$ \\ Department of Experimental Oncology, European Institute of Oncology, 20141 Milan, Italy
}

Despite the fact that ovarian cancer is the leading cause of death from gynecological disease in American women leading to an estimated number of more than 14,000 deaths in 2003 (American Cancer Society 2003), the molecular events occurring during ovarian tumorigenesis have not been unraveled in great detail. The discovery of these molecular events has proven particularly difficult for ovarian cancer, in part because this type of cancer is often discovered at late stages, limiting the availability of material for studies of the initiating events in ovarian cancer (Cvetkovic 2003). The pursuit of genes critical for ovarian tumorigenesis has nonetheless revealed a number of genes that may be implicated. Loss of heterozygosity (LOH) studies have established that chromosome 17 is a hotspot for chromosomal aberrations in breast (Phelan et al. 1998) and ovarian cancer (Eccles et al. 1992; Foulkes et al. 1993; Phillips et al. 1993, 1996b; Godwin et al. 1994; Saretzki et al. 1997; Wiper et al. 1998). Candidate oncogenes and tumor suppressors located on this chromosome include P53, BRCA1, HER2/NEU, NF1, NM23, HIC1, and the two recently discovered putative tumor suppressor genes OVCA1 and OVCA2 (for review, see Schuijer and Berns 2003; Fig. 1). P53 is the most frequently mutated gene in advanced epithelial ovarian carcinomas. However, in benign and borderline ovarian neoplasms, P53 mutations are relatively rare; suggesting that p53 loss-of-function is a late event in ovarian carcinogenesis (Godwin et al. 1997). The two breast cancer genes, $B R C A 1$ and $B R C A 2$, are often mutated in women with a family history of ovarian and breast cancer. However, $B R C A 1$ and $B R C A 2$ do not appear to be linked to the development of sporadic ovarian cancer, which accounts for the large majority of ovarian cancer cases. Recently, a novel interesting putative oncogene, EMSY, was found to be amplified in a large proportion (17\%) of late stage ovarian carcinomas (Hughes-Davies et al. 2003). Interestingly, EMSY amplification was only observed in breast and ovarian cancer. EMSY associates with BRCA2, and overexpression may imitate loss of BRCA2 in both sporadic breast and ovarian cancer. Because no cases of amplification were found in lower-

\footnotetext{
1 Corresponding author.

E-MAIL khelin@ieo.it; FAX 39-025-748-9851.

Article and publication are at http://www.genesdev.org/cgi/doi/10.1101/ gad.1184004
}

grade ovarian tumors, EMSY amplification is likely to be a late event in ovarian tumorigenesis. This unfortunately limits its use as a diagnostic marker for early detection. However, EMSY activation in concert with deactivation of tumor suppressor genes may be an important tumorigenic event. Further studies are needed to firmly establish EMSY as a significant ovarian oncogene.

\section{OVCA1 and OVCA2}

In recent years, the BRCA1 inducible (Atalay et al. 2002) ovarian cancer genes 1 and 2 (OVCA1 and OVCA2) have attracted some attention. These genes are located on human chromosome $17 \mathrm{p} 13.3$, which is deleted in $\sim 80 \%$ of ovarian cancer (Phillips et al. 1993, 1996a) and were isolated by positional cloning (Schultz et al. 1996). Although their names could suggest functional biological relationship, the sequences of the two proteins are, in fact, completely unrelated (Schultz et al. 1996; Chen and Behringer 2001). In addition, their amino acid sequences do not reveal their biological function, as neither of them contain regions with homology to known functional protein domains. Nevertheless, OVCA1 shares overall homology (about 20\% identity) with diphthamide biosynthesis protein 2 (DPH2) from Saccharomyces cerevisiae, and is also known as DPH2 like 1 (DPH2L1; Phillips et al. 1996a; Schultz et al. 1996). At least one additional member of this protein family exists. DPH2 like 2 (DPH2L2), which has been identified on human chromosome 1p34 (Schultz et al. 1998), could potentially supply functional redundancy to OVCA1.

Defining the precise biological and biochemical functional relevance of the OVCA genes with respect to ovarian cancer has proven rather difficult. The two OVCA genes are expressed from the same genetic locus using two different promoters; however, since the OVCA2 transcript contains a unique exon and only the 3' UTR of the OVCA1 transcript, they share no coding sequence (Schultz et al. 1996; Fig. 1 in Chen and Behringer 2004). In addition, data have suggested that several isoforms of the OVCA1 protein exist, which so far do not have any assigned function (Bruening et al. 1999). It is nonetheless known that both OVCA genes are ubiquitously expressed in normal human tissues and during various stages of development (Phillips et al. 1996a; Schultz et al. 1996; Prowse et al. 2002). Interestingly, significantly 
Jensen and Helin

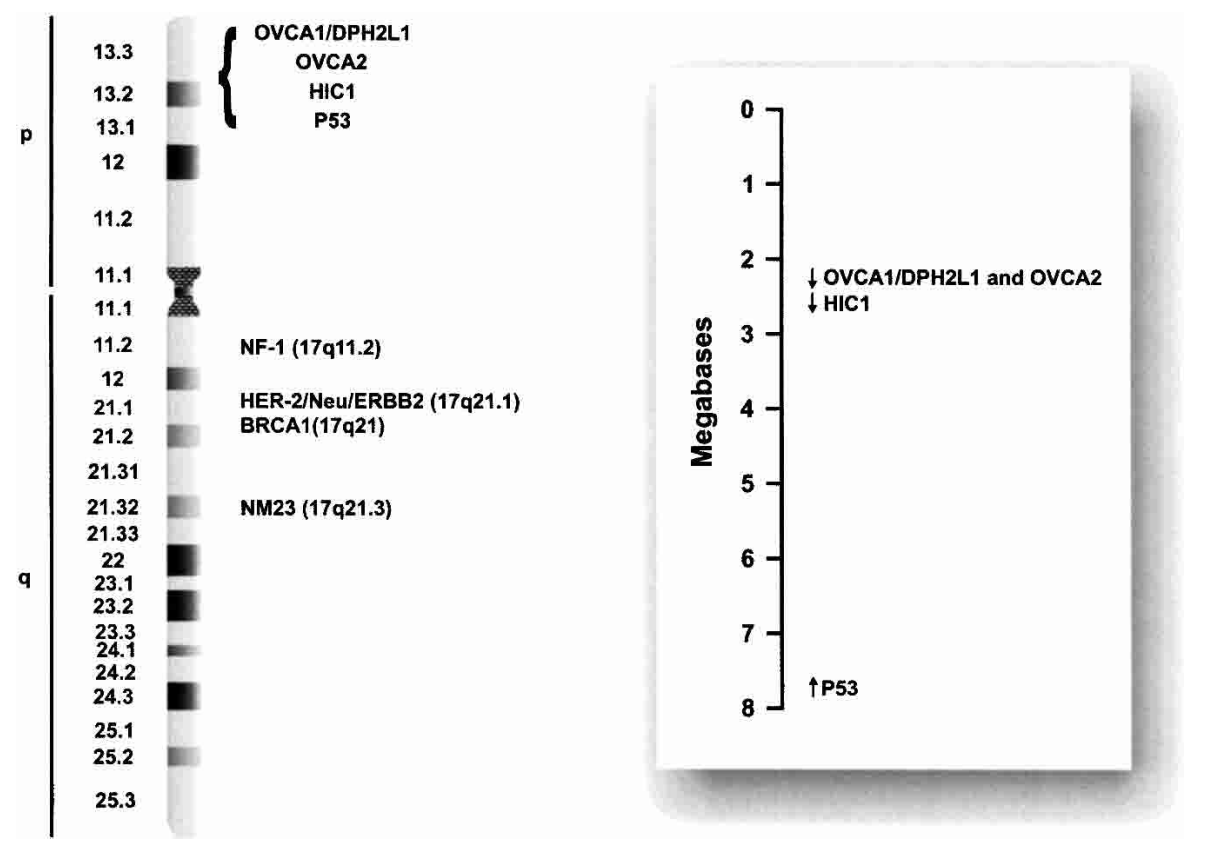

Figure 1. Potential tumor suppressors and oncogenes on human chromosome 17. OVCA1/DHP2L1, OVCA2, HIC1, and P53 are located within an $8-\mathrm{Mb}$ region on chromosome 17 . The genes are similarly linked on mouse chromosome 11 . Chromosome 17 contains several regions that are hotspots for alterations in cancer. In particular, a region (17p13.3) containing the putative tumor suppressors OVCA1, OVCA2, and HIC1 are deleted in $80 \%$ of ovarian cancer. In several cases, the deletion of $17 \mathrm{p} 13.3$ was not accompanied by the deletion of $17 \mathrm{p} 13.1$ containing P53, suggestion an independent tumor suppressive role for one or more of the three candidate genes. The study by Chen and Behringer (2004) suggests that the tumor suppressor gene is OVCA1, whereas a recent published paper by Baylin and colleagues (Chen et al. 2003) demonstrate that mice heterozygous for Hic1 are also tumor prone, developing a broad spectrum of tumors. Taken together, these results demonstrate that at least three tumor-suppressor genes are located within $8 \mathrm{Mb}$ of human chromosome 17. The loss-of-function of one or more of these three genes may determine the incidence, the phenotype, and the tissue in which tumors develop.

lower or absent OVCA1 expression was observed in ovarian tumor tissues or cell lines both at the mRNA and protein level (Schultz et al. 1996; Bruening et al. 1999), suggesting that OVCA1 loss may be an important event in ovarian tumorigenesis. In support of this hypothesis, Bruening et al. (1999) demonstrated that the consequence of OVCA1 overexpression in A2780 ovarian cancer cells is suppression of growth with an increased number of cells in the $G_{1}$ phase of the cell cycle. As such, OVCA1 appears to play an as yet ill-defined role in cell cycle/cell growth control.

\section{Embryonic lethality of Ovca1-deficient mice}

To address the role of Ovca1 and Ovca2 in development and proliferation, Chen and Behringer (2004) generated two novel, genetically modified mouse models in which Ovca1 has been disrupted either alone or in combination with Ovca2. Because Ovca1 and Ovca2 are derived from overlapping transcript, it has been complicated to determine which of the genes (or both) plays a major role in ovarian tumorigenesis. The results that are presented suggest that OVCA1 is the more important gene in the locus with respect to proliferation and tumorigenesis, as identical results are obtained from both strains of mice.

First, Chen and Behringer (2004) demonstrated that
Ovca1 is essential for normal mouse development, suggesting that the homologous DPH2L2 gene (Schultz et al. 1998) cannot substitute for OVCA1 gene function. Embryos lacking Ovca1 do not show any gross abnormalities; however, they appear to be delayed $\sim 1 \mathrm{~d}$ in development. This developmental delay results in Ovca1 null embryos that die between midgestation and birth. The Ovca1-deficient embryos weigh about half of their normal or heterozygous siblings. These results are consistent with the idea that OVCA1 is a regulator of cell proliferation. A number of specific developmental defects were observed in the mice, including invariably cleft palate and an immature lung phenotype-possibly the main cause of death for newborns.

To further evaluate the effects of Ovca1 loss on proliferation, Chen and Behringer (2004) derived mouse embryonic fibroblasts (MEFs). Ovcal-deficient MEFs grow more slowly and accumulate a larger proportion of cells in the $G_{1}$ phase of the cell cycle, consistent with a reduced phosphorylation of the retinoblastoma protein, pRB. These results could suggest that OVCA1 is a positive regulator of cell proliferation, or alternatively, that loss of OVCA1 could lead to activation of a cell cycle checkpoint. P53 is a key regulator of cell cycle checkpoints, and as the OVCA locus is cytogenetically close to $P 53$ in mice and humans, and deletion of both loci are 
often seen in human tumors, Chen and Behringer (2004) tested whether loss of p53 would rescue the proliferation defect seen in the Ovca-deficient MEFs. Even though the OVCA and P53 loci are located only $\sim 8 \mathrm{cM}$ apart (Fig. 1), mice with a spontaneous chromosomal recombination event were obtained, and the proliferation phenotype of Ovca1 null MEFs appeared to be completely rescued by additional deletion of p53. However, despite the rescue of proliferation, loss of $P 53$ was not sufficient to prevent the lethality of the Ovca1-deficient embryos, although a partial rescue of the size and cleft palate phenotypes were observed. These data may suggest that a checkpoint is induced in the mouse embryo fibroblasts, which is dependent on p53; however, it is not only a p53-dependent checkpoint that results in lack of proliferation in Ovca1-deficient mouse embryos.

\section{Ovca1 heterozygous mice are tumor prone}

One of the key objectives of this study was to determine whether Ovca1 acts as a tumor suppressor in vivo. Data obtained by xenografting ovaries from Ovca1 null embryos were disappointing, as no ovarian tumors developed within 9 mo. In contrast, Ovca1-deficient ovaries grew more poorly than the wild-type controls. Although these data correlated well with the proliferative deficiency and developmental delay observed in MEFs and embryos, they do not present evidence for Ovca1 being a tumor suppressor.

The heterozygous Ovca1 mice and various genotypes generated by crosses with p53-deficient mice were followed for 2 yr to study spontaneous tumorigenesis. Interestingly, Ovca1 heterozygous mice are tumor prone with $>50 \%$ of the mice developing a variety of tumors; a rate that is comparable with the tumor incidence observed in P53 heterozygous mice. However, the time of tumor onset is significantly later in Ovca1 heterozygotes (about $12 \mathrm{wk}$, mean latency of $92 \mathrm{wk}$ ) compared with p53 heterozygous mice. p53 null mice spontaneously develop tumors so early that concomitant loss of one Ovca1 allele has no apparent effect on tumorigenic rate or incidence. However, Ovca1 deletion leads to earlier lethality and tumor development in a p53 heterozygous background.

Because LOH of the OVCA1/2 locus is a frequent event in at least late stage ovarian cancers, the Ovca1 null mice were anticipated to acquire ovarian lesions of benign and/or malignant nature. This turned out to be only partially true. Although one benign ovarian tumor (specifically a serous cystadenoma) was found in a 1-1/2year-old mouse, the majority of the tumors arose in various other tissues. These data are, in part, consistent with the lack of ovarian tumor formation in xenografts from Ovca1 null embryos. Interestingly, the wide spectrum of tumors, which arose in the Ovca1 heterozygous mice, was different from what has been described for p53 heterozygous mice, and the variety of tumors was larger in the double heterozygous mice compared with the individual heterozygotes.

\section{Biochemical function of OVCA1?}

The surprising lack of correlation between the number of ovarian tumors in Ovca1 heterozygous mice and deletions in human ovarian tumors is difficult to explain, especially when so little is known about the biochemical function the OVCA proteins. As mentioned previously, ectopic Ovcal overexpression in various cell lines (Bruening et al. 1999), as well as complete loss in MEFs, leads to some sort of growth suppression. Generally, loss of a tumor suppressor gene leads to increased proliferation of MEFs (e.g., p53, p2 $7^{\text {Kip1 }}, \mathrm{p} 16^{\text {INK4a }}$, and $\mathrm{p} 19^{\text {Arf }}$, although this effect tends to be cell-type specific due to tissue-specific modifier genes. However, in the case of Ovca1, loss of one allele in the mouse leads to tumor development, whereas loss of both alleles results in embryonic lethality. It is generally believed that patients with a monoallelic mutation in a tumor suppressor gene (e.g., P53, RB1, or BRCA1) are predisposed to cancer, because loss of the healthy allele will lead to manifestation of disease. However, it is not clear whether the second allele is lost in the tumors appearing in the Ovca1 heterozygous mice. It is possible that this is a case of haploinsufficiency, in which low Ovcal expression in combination with loss of other tumor suppressors or overexpression of oncogenes is involved in tumorigenesis. How could this occur? Do low Ovcal levels allow or even predispose for additional mutations, whereas complete loss leads to death? Ovca1 is induced by BRCA1 (Atalay et al. 2002), which is known to play an important role in DNA damage repair (for review, see Lou and Chen 2003; Powell and Kachnic 2003). On the basis of this observation and the fact that the growth defect of Ovcal deficient MEFs is rescued by concomitant loss of p53, it is tempting to speculate that Ovcal could be part of a DNA damage-control system. However, the connection between Ovcal and p53 remains to be established. Ovca1-deficient MEFs do not have increased levels of the p53 target gene, p21, nor do they undergo extensive apoptosis. Similarly, the authors did not find evidence for increased levels of apoptosis or decreased proliferation in Ovca1 null embryos as compared with wild-type or heterozygous littermates, suggesting that if Ovcal is involved in a DNA damage checkpoint, the checkpoint is different from those described so far. The generation of specific antibodies to Ovcal, together with the availability of the Ovca1-deficient mice, should allow the elucidation of the biochemical function of Ovcal. Furthermore, to test the function of Ovcal in adult mice, it would be interesting to create conditional Ovca1 knockout mice. Would these mice die immediately (and from what) or develop tumors at an increased rate?

Chen and Behringer's study (2004) is a major step toward understanding the genetic complexity of not only ovarian, but also other types of cancer. This study shows convincingly that loss of OVCA1 can contribute to tumorigenesis. Because specific deletions of the locus have been found in human tumors, these data are the first to demonstrate that OVCA1 is a bona fide tumor-suppressor gene. Further studies using additional mouse models 
in which combinations of tumor-suppressor genes frequently lost in cancer are mutated, will have major impact on our understanding of the complex nature of the multiple events occurring, which eventually leads to ovarian cancer.

\section{Acknowledgments}

The work in the authors' laboratory is supported by grants from the Association for International Cancer Research (AICR), Associazione Italiana per la Ricerca sul Cancro (AIRC), Fondazione Italiana per la Ricerca sul Cancro (FIRC), The Italian Health Ministry, and the European Union.

\section{References}

American Cancer Society. 2003. Cancer facts and figures 2003. American Cancer Society, Inc., Atlanta, GA.

Atalay, A., Crook, T., Ozturk, M., and Yulug, I.G. 2002. Identification of genes induced by BRCA1 in breast cancer cells. Biochem. Biophys. Res. Commun. 299: 839-846.

Bruening, W., Prowse, A.H., Schultz, D.C., Holgado-Madruga, M., Wong, A., and Godwin, A.K. 1999. Expression of OVCA1, a candidate tumor suppressor, is reduced in tumors and inhibits growth of ovarian cancer cells. Cancer Res. 59: 4973-4983.

Chen, C.M. and Behringer, R.R. 2001. Cloning, structure, and expression of the mouse Ovcal gene. Biochem. Biophys. Res. Commun. 286: 1019-1026.

- 2004. Ovca1 regulates cell proliferation, embryonic development, and tumorigenesis. Genes \& Dev. (this issue).

Chen, W.Y., Zeng, X., Carter, M.G., Morrell, C.N., Chiu Yen, R.W., Esteller, M., Watkins, D.N., Herman, J.G., Mankowski, J.L., and Baylin, S.B. 2003. Heterozygous disruption of Hicl predisposes mice to a gender-dependent spectrum of malignant tumors. Nat. Genet. 33: 197-202.

Cvetkovic, D. 2003. Early events in ovarian oncogenesis. Reprod. Biol. Endocrinol. 1: 68.

Eccles, D.M., Brett, L., Lessells, A., Gruber, L., Lane, D., Steel, C.M., and Leonard, R.C. 1992. Overexpression of the p53 protein and allele loss at $17 \mathrm{p} 13$ in ovarian carcinoma. Br. J. Cancer 65: 40-44.

Foulkes, W.D., Black, D.M., Stamp, G.W., Solomon, E., and Trowsdale, J. 1993. Very frequent loss of heterozygosity throughout chromosome 17 in sporadic ovarian carcinoma. Int. J. Cancer 54: 220-225.

Godwin, A., Schultz, D., Hamilton, T., and Knudson, A. 1997. Oncogenes and tumor suppressor genes. In Principles and practice of gynecological oncology (ed. W. Hoskins, C. Perez, and R. Young), pp. 107-148. J.B. Lippincott Co., Philadelphia, PA.

Godwin, A.K., Vanderveer, L., Schultz, D.C., Lynch, H.T., Altomare, D.A., Buetow, K.H., Daly, M., Getts, L.A., Masny, A., Rosenblum, N., et al. 1994. A common region of deletion on chromosome $17 \mathrm{q}$ in both sporadic and familial epithelial ovarian tumors distal to BRCA1. Am. I. Hum. Genet. 55: 666-677.

Hughes-Davies, L., Huntsman, D., Ruas, M., Fuks, F., Bye, J., Chin, S.F., Milner, J., Brown, L.A., Hsu, F., Gilks, B., et al. 2003. EMSY links the BRCA2 pathway to sporadic breast and ovarian cancer. Cell 115: 523-535.

Lou, Z. and Chen, J. 2003. BRCA proteins and DNA damage checkpoints. Front. Biosci. 8: s718-s721.

Phelan, C.M., Borg, A., Cuny, M., Crichton, D.N., Baldersson,
T., Andersen, T.I., Caligo, M.A., Lidereau, R., Lindblom, A., Seitz, S., et al. 1998. Consortium study on 1280 breast carcinomas: Allelic loss on chromosome 17 targets subregions associated with family history and clinical parameters. Cancer Res. 58: 1004-1012.

Phillips, N., Ziegler, M., Saha, B., and Xynos, F. 1993. Allelic loss on chromosome 17 in human ovarian cancer. Int. J. Cancer 54: 85-91.

Phillips, N.J., Zeigler, M.R., and Deaven, L.L. 1996a. A cDNA from the ovarian cancer critical region of deletion on chromosome 17p13.3. Cancer Lett. 102: 85-90.

Phillips, N.J., Ziegler, M.R., Radford, D.M., Fair, K.L., Steinbrueck, T., Xynos, F.P., and Donis-Keller, H. 1996b. Allelic deletion on chromosome $17 \mathrm{p} 13.3$ in early ovarian cancer. Cancer Res. 56: 606-611.

Powell, S.N. and Kachnic, L.A. 2003. Roles of BRCA1 and BRCA2 in homologous recombination, DNA replication fidelity and the cellular response to ionizing radiation. Oncogene 22: 5784-5791.

Prowse, A.H., Vanderveer, L., Milling, S.W., Pan, Z.Z., Dunbrack, R.L., Xu, X.X., and Godwin, A.K. 2002. OVCA2 is downregulated and degraded during retinoid-induced apoptosis. Int. J. Cancer 99: 185-192.

Saretzki, G., Hoffmann, U., Rohlke, P., Psille, R., Gaigal, T., Keller, G., Hofler, H., Loning, T., Petersen, I., and Dietel, M. 1997. Identification of allelic losses in benign, borderline, and invasive epithelial ovarian tumors and correlation with clinical outcome. Cancer 80: 1241-1249.

Schuijer, M. and Berns, E.M. 2003. TP53 and ovarian cancer. Hum. Mutat. 21: 285-291.

Schultz, D.C., Vanderveer, L., Berman, D.B., Hamilton, T.C., Wong, A.J., and Godwin, A.K. 1996. Identification of two candidate tumor suppressor genes on chromosome $17 \mathrm{p} 13.3$. Cancer Res. 56: 1997-2002.

Schultz, D.C., Balasara, B.R., Testa, J.R., and Godwin, A.K. 1998. Cloning and localization of a human diphthamide biosynthesis-like protein-2 gene, DPH2L2. Genomics 52: 186191.

Wiper, D.W., Zanotti, K.M., Kennedy, A.W., Belinson, J.L., and Casey, G. 1998. Analysis of allelic imbalance on chromosome $17 \mathrm{p} 13$ in stage I and stage II epithelial ovarian cancers. Gynecol. Oncol. 71: 77-82. 


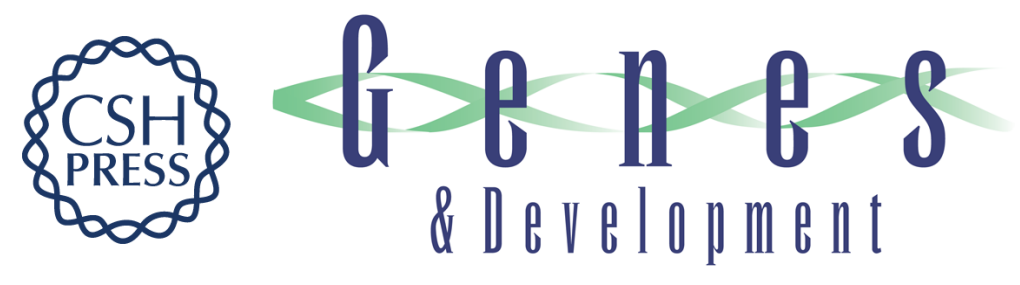

\section{OVCA1: emerging as a bona fide tumor suppressor}

Michael Rugaard Jensen and Kristian Helin

Genes Dev. 2004, 18:

Access the most recent version at doi:10.1101/gad.1184004

References This article cites 21 articles, 4 of which can be accessed free at: http://genesdev.cshlp.org/content/18/3/245.full.html\#ref-list-1

License

Email Alerting Receive free email alerts when new articles cite this article - sign up in the box at the top Service right corner of the article or click here.

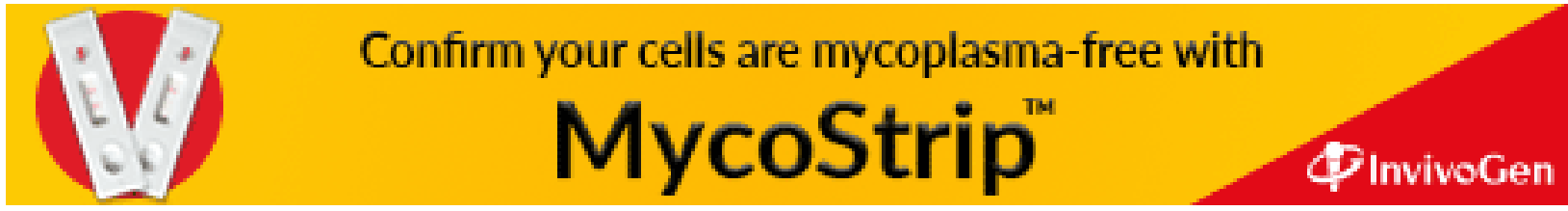

